Revista Científica do Corpo de Bombeiros Militar de Pernambuco

XVIII Seminário Nacional de Bombeiros - Foz do Iguaçu PR

Vol.04 No11 - Edição Especial XVIII SENABOM - ISSN 2359-4829

Versão on-line disponível em: http://www.revistaflammae.com.

\title{
RELAÇÃO ENTRE OS EVENTOS DE EL NINÕ E LA NINÃ COM OS DECRETOS DE SITUAÇÃO DE EMERGÊNCIA E ESTADO DE CALAMIDADE PÚBLICA PARA O ESTADO DO RIO GRANDE DO SUL
}

\author{
COMPARISON BETWEEN THE EVENTS OF EL NIÑO AND LA NINA WITH THE \\ DECREES OF THE STATE OF EMERGENCY AND STATE OF PUBLIC CALAMITY \\ FOR THE STATE OF RIO GRANDE DO SUL
}

\author{
Elison Eduardo Jardim Bierhals ${ }^{1}$ \\ Claudinéia Machado Brazil ${ }^{2}$ \\ Marcelo Carvalho Soares ${ }^{3}$
}

\section{Resumo}

O Corpo de Bombeiros Militar tem um papel fundamental frente às demandas de ocorrências de desastres naturais, principalmente no que tange a resposta operacional em busca e resgaste. $\mathrm{O}$ Rio Grande do Sul, tem registrado diversos eventos severos associados a escassez de água e de grande instabilidade atmosférica, que são desastres com potencial de pronta resposta. Devido ao exposto, esse trabalho tem como objetivo verificar a influência das alterações de temperatura da superfície do mar (TSM), nos desastres que ocasionaram situação de emergência ou estado de calamidade pública, decretados pelos municípios e reconhecidos pela Defesa Civil Nacional. Os resultados apontaram que as anomalias de TSM exerceram influência na ocorrência desses eventos. Espera-se que esse trabalho sirva como subsidio para oferecer suporte aos sistemas especializados de alertas, auxiliando nas operações relacionadas a ocorrência de desastres no Estado do Rio Grande do Sul.

Palavras-chave: desastres naturais; situação de emergência; La Niña; El Niño.

\section{Abstract}

The Military Fire Brigade has a fundamental role to face the demands of occurrences of natural disasters, mainly regarding the operational response in search and rescue. Rio Grande do Sul has recorded several severe events associated with water scarcity and great atmospheric instability, which are disasters with the potential for prompt response. Due to the above, this work has the objective of verifying the influence of changes in sea surface temperature (SST), in the disasters that caused an emergency or a state of public calamity, decreed by the municipalities and recognized by the National Civil Defense. The results showed that SST anomalies influenced the occurrence of these events. It is hoped that this work will serve as a subsidy to support the specialized alert systems, assisting in operations related to the occurrence of disasters in the State of Rio Grande do Sul.

Key words: disasters; emergency situation; La Niña; El Niño.

\footnotetext{
${ }^{1}$ Corpo de Bombeiros Militar do Rio Grande do Sul, graduando Engenharia de Energia, bierhalseduardo@gmail.com

${ }^{2}$ Faculdade Dom Bosco de Porto Alegre, Doutora, neiabrazil@gmail.com

${ }^{3}$ Corpo de Bombeiros Militar do Rio Grande do Sul, Especialista, mcsoares.193@gmail.com
} 
Revista Científica do Corpo de Bombeiros Militar de Pernambuco

XVIII Seminário Nacional de Bombeiros - Foz do Iguaçu PR

Vol.04 Nº11 - Edição Especial XVIII SENABOM - ISSN 2359-4829

Versão on-line disponível em: http://www.revistaflammae.com.

\section{INTRODUÇ̃̃O}

O conhecimento dos fenômenos climáticos e dos desastres naturais a que nosso território está sujeito é fundamental para a efetividade de uma política de redução de riscos, objetivo primordial da Política Nacional de Proteção e Defesa Civil (Atlas, 2012). Os eventos climáticos extremos podem ocorrer de muitas formas diferentes, por exemplo, como inundações, secas, rajadas de vento, granizo, entre outros.

Durante muitos séculos, a humanidade tem desenvolvido uma boa compreensão da frequência de eventos climáticos extremos e as localizações geográficas onde eles são mais prováveis de acontecer (Santos, et. al. 2016). A ocorrência destes fenômenos meteorológicos de forma intensa constitui um motivo de preocupação para a sociedade, pois oferece alto potencial de destruição, perdas de vidas humanas e bens materiais, abalando direta ou indiretamente sua economia (Jiang et al., 2011).

Alterações na Temperatura da Superfície do Mar (TSM) exercem grande influência na variabilidade do comportamento climático. As condições oceânicas e atmosféricas sobre o Oceano Atlântico Tropical influenciam fortemente na variabilidade interanual do clima sobre as Américas (Ronchail et al., 2005).

As grandes anomalias de precipitação observadas na região nordeste, sul e sudeste do Brasil estão associadas a processos de interação oceano-atmosfera, tanto em escala global, quanto regional, em particular na Bacia do Atlântico. Entretanto, a TSM é apenas um dos fatores que influenciam o clima, sendo que a variação nas condições climáticas resulta de sua soma com outros fatores, entre eles o fenômeno El NiñoOscilação Sul (ENOS), que é caracterizado por anomalias, positivas (El Niño) ou negativas (La Niña), de temperatura da superfície do mar (TSM) no Pacífico equatorial (Andreolli \& Kayano, 2005),

O ENOS tem um tempo de retorno irregular, além de envolver diferentes intensidades podendo também não ocorrer, como é o caso dos anos neutros. Por suas características acaba interferindo diretamente no comportamento das condições atmosféricas, desse modo, contribuem com a ocorrência de eventos extremos. Embora a 
Revista Científica do Corpo de Bombeiros Militar de Pernambuco

XVIII Seminário Nacional de Bombeiros - Foz do Iguaçu PR

Vol.04 No11 - Edição Especial XVIII SENABOM - ISSN 2359-4829

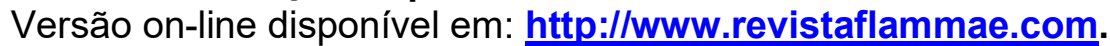

variação da TSM do Pacífico Equatorial apresente uma influência maior na variabilidade climática sobre a América do Sul, estudos mostram que a TSM do Atlântico Tropical Sul também exerce influência em algumas regiões Brasileiras (Andreoli \& Kayano, 2007).

No que tange a ocorrência de eventos meteorológicos severos, Kobiyama et al (2006), afirmam que os Estados da região Sul do Brasil têm sido austeramente impactados por estes, resultando em desastres naturais que ocasionaram grandes prejuízos socioeconômicos e ambientais, sendo que grande parte dos eventos estão associados às instabilidades severas que causam ventos fortes, granizos e inundações.

O Rio Grande do Sul, localizado nesta região, tem registrado diversos eventos severos associados à instabilidade intensa, no entanto, apresentando como características climáticas a ocorrências de chuvas bem distribuídas ao longo das quatro estações do ano. De acordo com Castro (2003) as inundações podem ocorrer de forma gradual ou de forma brusca, sendo a precipitação o fator determinante para o tipo de inundação.

As inundações bruscas são provocadas por chuvas intensas e concentradas, caracterizando-se por produzirem súbitas e violentas elevações nos níveis d'água dos corpos hídricos, e escoando de forma rápida e intensa. Muitas vezes, ocorrem associadas a áreas mais íngremes e em bacia de tamanho médio ou pequeno, sendo que a inclinação do terreno, ao favorecer o escoamento, contribui para intensificar a torrente e causar danos. Enquanto as inundações graduais estão relacionadas a longos períodos de chuva contínua e não intensa. Em síntese, verifica-se que este evento está diretamente relacionado à dinâmica fluvial que por sua vez está associada à precipitação.

Com relação ao granizo, sabe-se que é originado na parte superior das nuvens convectivas (cumulonimbus), que possuem elevado desenvolvimento vertical e temperaturas muito baixas no seu topo. Tais condições são propícias para transformar as gotículas de água em partículas de gelo que se movimentam com as correntes 
subsidentes e ao se chocarem com gotas d'águas mais frias, aumentam seu tamanho, alcançando dimensões de queda, ou seja, tornam-se grandes em demasia para serem sustentados pelas correntes ascendentes ocorrendo, assim, a precipitação de granizo (Andreoli et al., 2007).

Os ventos fortes, conforme Castro (1999), consistem no deslocamento violento de uma massa de ar de uma área de alta pressão para uma área de baixa pressão. Reckiziegel, (2007) contribui informando que a ocorrência de vendavais, muitas vezes está associada à passagem de frentes frias, sendo que sua força será tanto maior quanto maior for a diferença de pressão das massas de ar em frontogênese.

De acordo com De Castro (1999) os desastres podem ser classificados quanto à evolução como: súbitos ou de evolução aguda, graduais ou de evolução cônica e por somação de efeitos parciais. Os desastres súbitos ou de evolução aguda apresentam uma potencial necessidade de intervenção de pronta resposta ou de emergência por parte dos órgãos públicos como Corpo de Bombeiros e de Defesa Civil, por esse motivo os decretos foram separados em eventos com necessidade de pronta resposta ou necessidade resposta ordinária por parte das autoridades competentes.

Os eventos extremos que geram situações de emergência ou estado de calamidade pública, decretados por seus respectivos governos municipais, de acordo com suas características, podem ou não ter esses decretos reconhecidos pela Defesa Civil, os dados de desastres naturais utilizados nesta pesquisa, foram somente os reconhecidos pela Secretaria Nacional de Defesa Civil.

A Emenda Constitucional n ${ }^{\circ}$ 67, de 17/06/14 em seu art 130 e o consequente desmembramento do Corpo de Bombeiros Militar, da Brigada Militar, assim ficou definido:

Art. 130. Ao Corpo de Bombeiros Militar, dirigido pelo (a) Comandante Geral, oficial (a) da ativa do quadro de Bombeiro Militar, do último posto da carreira, de livre escolha, nomeação e exoneração pelo (a) Governador (a) do Estado, competem a prevenção e o combate de incêndios, as buscas e salvamentos, as ações de defesa civil e a polícia judiciária militar, na forma definida em lei complementar. (RIO GRANDE DO SUL, 2014). 
Este dispositivo legal é um dos que defini como atribuição do Corpo de Bombeiros do Estado do Rio Grande do sul a atividade de Defesa Civil, atividade para a qual pode ser útil o conhecimento da relação do ENOS com os desastres naturais.

Este artigo tem como objetivo estudar os padrões associados entre os eventos meteorológicos extremos, que atingiram o Estado do Rio Grande do Sul, ocasionando situação de emergência ou estado de calamidade pública, decretados pelos municípios e reconhecidos pela Defesa Civil Nacional e a relação destes desastres com as anomalias da TSM, buscando conhecer as características espaço temporal desses eventos que causam danos à sociedade. Uma das missões do Corpo de Bombeiros, através de ações de defesa civil, é viabilizar o retorno da população atingida por desastres para sua ocupação original.

\section{DESENVOLVIMENTO}

O Rio Grande do Sul está localizado no extremo meridional do Brasil. Em razão de sua posição latitudinal, seu clima apresenta estações bem definidas ao longo do ano, sofrendo grande influência de sistemas de latitudes médias, sendo os sistemas frontais os mais frequentes causadores de chuvas durante o ano todo (Oliveira, 1986).

Figura 1- Mapa Localização da Região de Estudo
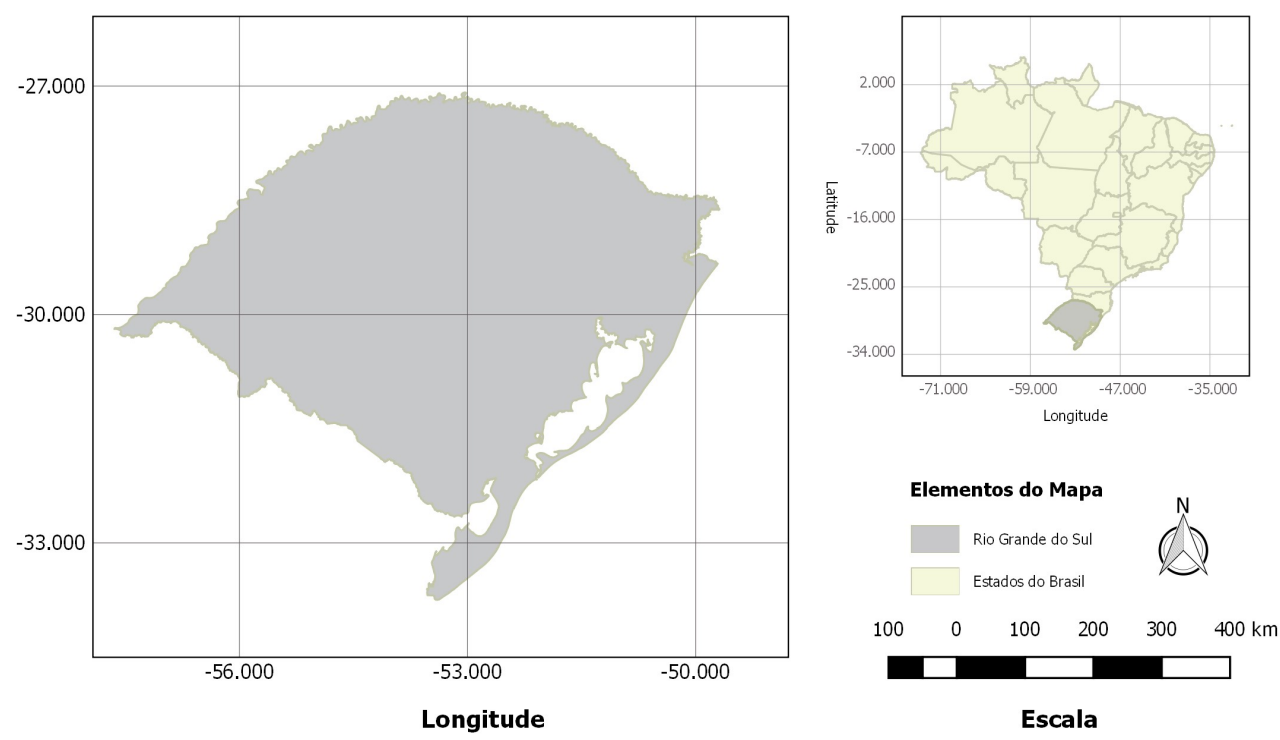


\section{Revista FLAMMAE}

Revista Científica do Corpo de Bombeiros Militar de Pernambuco

XVIII Seminário Nacional de Bombeiros - Foz do Iguaçu PR

Vol.04 No11 - Edição Especial XVIII SENABOM - ISSN 2359-4829

Versão on-line disponível em: http://www.revistaflammae.com.

\section{Dados e Metodologia}

Os decretos de ocorrência dos eventos extremos foram coletados no site do sistema integrado de informações aos desastres e compreendem o período de 2003 a 2016. Os dados de Temperatura da Superfície do Mar (TSM) das regiões dos Niños e Oceano Atlântico (ATL) foram obtidos junto ao National Oceanic and Atmospheric Administration (NOAA). A Figura 2 apresenta as regiões que foram utilizadas: Niño $1+2\left(0^{\circ}-10^{\circ} \mathrm{S} ; 80^{\circ}-90^{\circ} \mathrm{W}\right)$; Niño $3\left(5^{\circ} \mathrm{S}-5^{\circ} \mathrm{N} ; 90^{\circ}-150^{\circ} \mathrm{W}\right)$; Niño $3.4\left(5^{\circ} \mathrm{S}-5^{\circ} \mathrm{N} ; 120^{\circ}-\right.$ $\left.170^{\circ} \mathrm{W}\right)$, Niño $4\left(5^{\circ} \mathrm{S}-5^{\circ} \mathrm{N} ; 160^{\circ} \mathrm{E}-150^{\circ} \mathrm{W}\right)$, Atlântico Norte $\left(5-20^{\circ} \mathrm{N}, 60-30^{\circ} \mathrm{W}\right)$, Attântico Tropical Sul $\left(0-20^{\circ} \mathrm{S}, 30^{\circ} \mathrm{W}-10^{\circ} \mathrm{E}\right)$ e Equatorial Global $\left(10^{\circ} \mathrm{S}-10^{\circ} \mathrm{N}, 0^{\circ}-360^{\circ}\right)$.

Figura 2 - Regiões de Temperatura da Superfície do Mar (TSM).

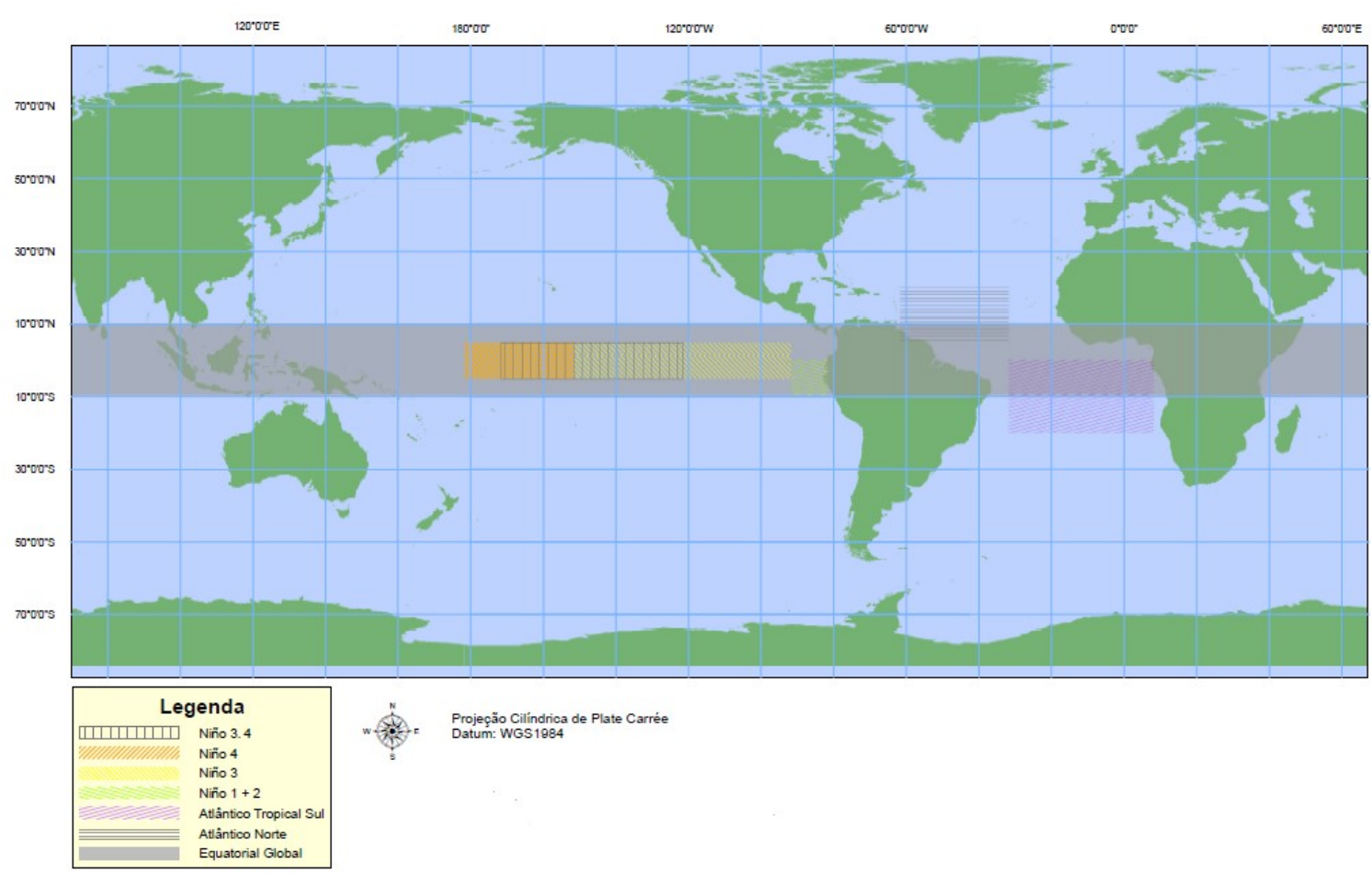

A análise da ocorrência de eventos extremos e sua variabilidade foram realizadas comparando os dados obtidos junto a Defesa Civil e os dados de TSM na região Niño 3.4. Para encontrar relações que possam descrever as conexões dos diferentes índices climáticos com a série de eventos extremos no Rio Grande do Sul, os dados obtidos junto a defesa civil, foram organizados de forma a possibilitar uma análise dos decretos e sua relação com as anomalias de TSM. 


\section{RESULTADOS}

A ocorrência de desastres na região Sul do Brasil está diretamente relacionada aos altos índices de precipitação, causando as inundações que ainda podem ser agravadas por outros fenômenos como ventos fortes, granizo, entre outros (Teixeira \& Satyamurty, 2004). Portanto, nesta seção foram avaliadas a relação dos eventos extremos com o fenômeno ENOS para verificar se existe uma relação entre esses fenônemos e a ocorrência de desastres que ocasionaram SE/ECP.

A figura 3 apresenta o número de ocorrência de eventos extremos no estado do Rio Grande do Sul com decreto de SE/ECP reconhecidos pela Secretaria Nacional de Defesa Civil, em anos de El Niño, La Niña e Neutros de 2003 a 2016, sendo este último representante dos anos sem a influência do ENOS (El Nino Oscilação Sul).

Analisando o gráfico (figura 3) podemos observar que no período avaliado os decretos de SE/ECP em função de eventos de estiagem e/ou seca aumentaram quando o estado esteve sobre influência do El Niño chegando próximo a 850 (700 estiagens e 150 secas), enquanto nos anos neutros esse número foi de aproximadamente 300 decretos e já nos anos sob influência do La Niña os decretos de SE/ECP em ficaram abaixo de 600 .

O Estado do Rio Grande do sul possui 639 registros oficiais de enxurradas reconhecidas pela Defesa Civil Nacional, entre 2003 a 2016, sendo que em torno de $65 \%$ dessas ocorrências foram sob influência do La Niña e $15 \%$ nos anos de El Niño e os anos neutros tiveram cerca de $20 \%$ dos casos de enxurradas.

Os eventos de Vendaval e enchentes foram mais registrados em períodos de influência do La Niña, sendo em torno de 100 decretos caracterizados por enchentes e quase 200 vendavais oficialmente reconhecidos, enquanto durante a influência do El Niño o número de enchentes não foi significante e os vendavais ficaram em torno de 110. 
Revista Científica do Corpo de Bombeiros Militar de Pernambuco

XVIII Seminário Nacional de Bombeiros - Foz do Iguaçu PR

Vol.04 No11 - Edição Especial XVIII SENABOM - ISSN 2359-4829

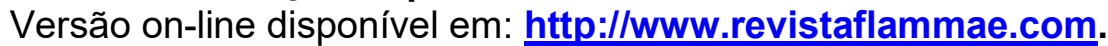

Figura 3 - Ocorrência de decretos de SE e ECP por tipo de evento (2003 - 2016)

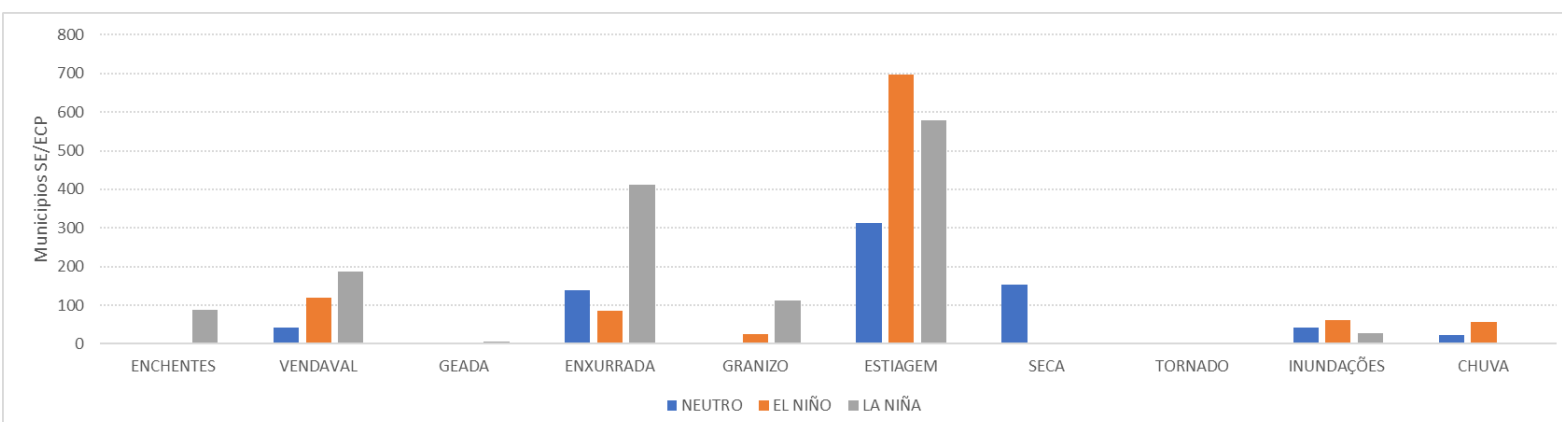

A tabela 1 demonstra a ocorrência de decretos de SE/ECP anual com e sem influência do ENOS. Os anos que apresentam a influência dos dois tipos de fenômenos (El Niño e La Niña) estão repetidos para melhor visualização de cada um. Os eventos estão separados por tipo de desastre de acordo com critério utilizado pela Defesa Civil.

Tabela 1 - Decretos anuais de SE/ECP por tipo de evento

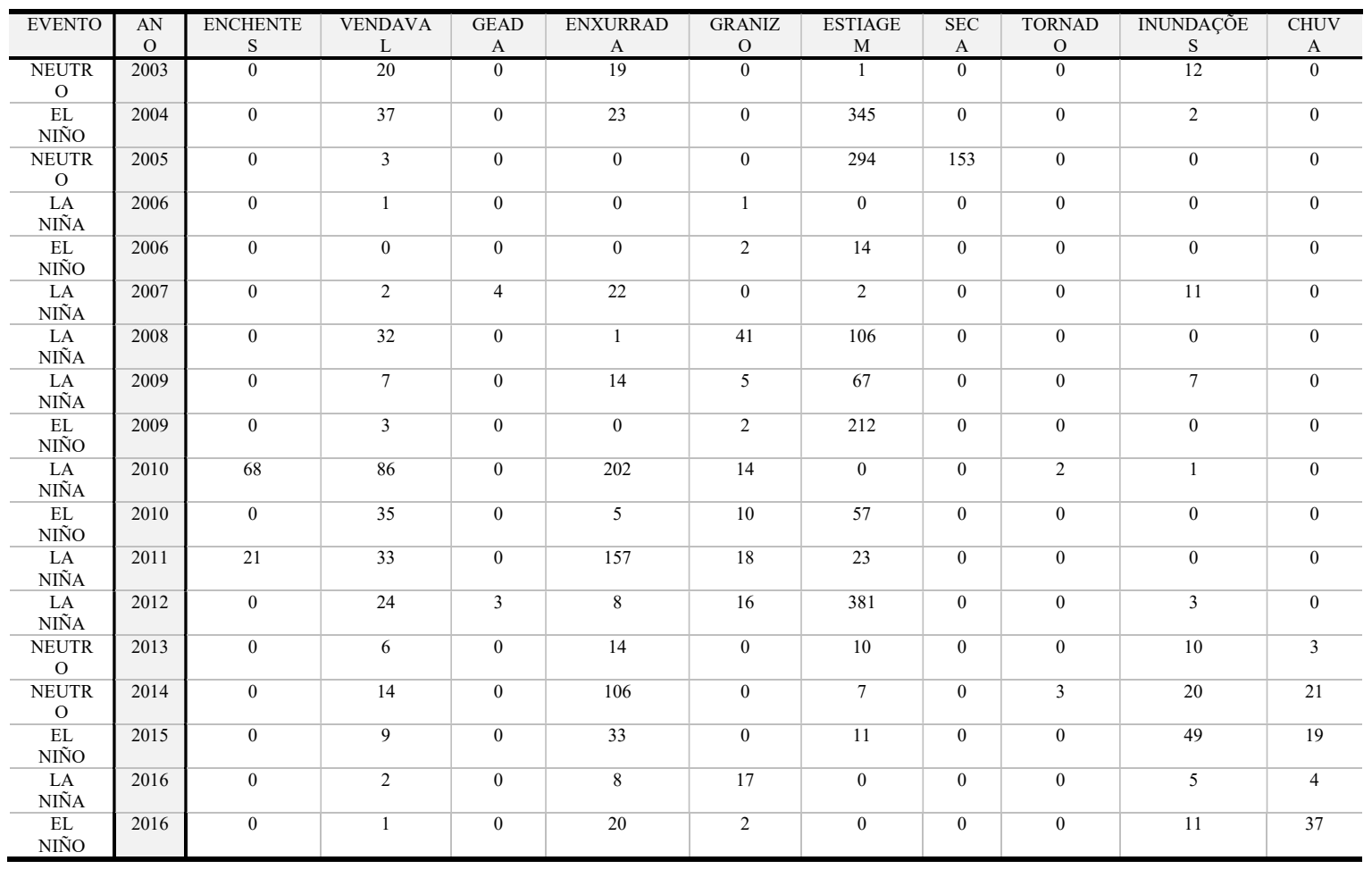

A figura 4 apresenta a relação do ENOS com os desastres que têm uma evolução súbita ou aguda, com potencial de intervenção de pronta resposta, pode-se 
Revista Científica do Corpo de Bombeiros Militar de Pernambuco

XVIII Seminário Nacional de Bombeiros - Foz do Iguaçu PR

Vol.04 N011 - Edição Especial XVIII SENABOM - ISSN 2359-4829

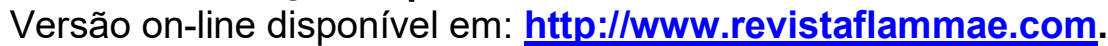

observar que durante a influência do La Niña a ocorrência de desastres que necessitam de uma resposta mais rápida por parte das autoridades é mais significante chegando a 800 desastres no período estudado, enquanto durante o El Niño a maioria dos desastres tem características de evolução mais gradual. Durante os anos sem influência do ENOS, observou-se que houve uma queda em ambos tipos de desastres.

Figura 4 - Ocorrência de decretos de SE e ECP por potencial de intervenção (2003 - 2016)

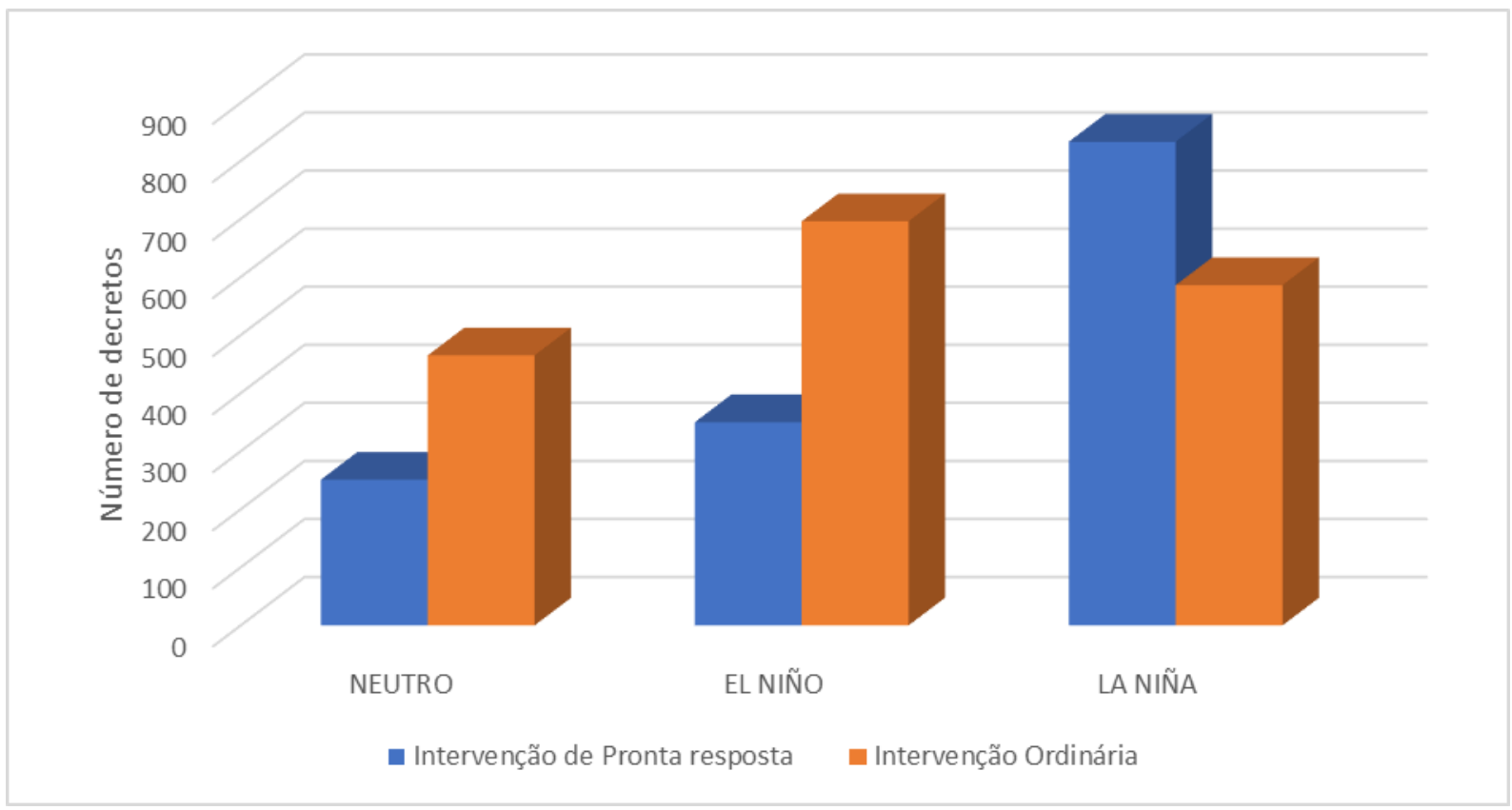

\section{CONCLUSÕES}

O entendimento da relação dos processos de interação oceano-atmosfera com os desastres naturais é fundamental para auxiliar o poder público na elaboração de medidas preventivas, planejamento de ações futuras e gestão dos territórios. A partir da análise dos resultados foi possível concluir que as anomalias de temperaturas da superfície do Oceano Pacífico exerceram um papel fundamental na ocorrência de eventos extremos no estado do Rio Grande do Sul, em especial na fase fria do ENOS.

O La Niña provocou um maior número de desastres de evolução aguda que tendem a causar maior destruição e danos em um curto espaço de tempo, e por 
Revista Científica do Corpo de Bombeiros Militar de Pernambuco

XVIII Seminário Nacional de Bombeiros - Foz do Iguaçu PR

Vol.04 N011 - Edição Especial XVIII SENABOM - ISSN 2359-4829

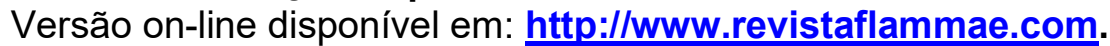

consequência com provável aumento na demanda de recursos à administração pública estadual visto a recorrente necessidade de intervenção de pronta resposta.

O desenvolvimento de trabalhos que visam ampliar conhecimentos referentes à temática é de suma importância pois ao passo que produzem uma metodologia de maior confiança, auxiliam às sociedades e também o poder público nas tomadas de decisões a serem empregadas, minimizando, assim, os impactos humanos e econômicos locais.

Como sugestão de trabalhos futuros, pode ser desenvolvida uma análise dos atendimentos e ocorrências por parte das unidades do Corpo de Bombeiros e sua relação com o ENOS.

\section{REFERÊNCIAS}

ANDREOLI, Rita Valéria; KAYANO, Mary Toshie. Previsões de temperatura da superfície do mar no Atlântico Tropical usando análises de correlações canônicas. Revista Brasileira de Meteorologia, São José dos Campos, v. 20, n. 1, p. 8493, 2005.

A importância relativa do Atlântico Tropical Sul e Pacífico Leste na variabilidade de precipitação do Nordeste do Brasil. Revista Brasileira de Meteorologia, v. 22, n. 1, p. 63-74, 2007.

Atlas Brasileiro de Desastres Naturais 1991-2010. Volume Brasil. CEPED/UFSC. Florianópolis, 2012.

DO SUL, RIO GRANDE. Constituição Estadual de 1989. ALERGS. Disponível em:< http://www2. al. rs. gov. br/dal/LinkClick. aspx, 2018.

DE CASTRO, Antônio Luiz Coimbra. Manual de planejamento em defesa civil. Ministério da Integração Nacional, Secretaria Nacional de Defesa Civil, 1999.

DOS SANTOS, Carlos Antonio Costa; MELO, Maria Monalisa MS; DE BRITO, José Ivaldo Barbosa. Tendências de Índices de Extremos Climáticos para o Estado do Amazonas e suas Relações com a TSM dos Oceanos Tropicais. Revista Brasileira de Meteorologia, v. 31, n. 1, p. 1-10, 2016.

JIANG, Dejuan et al. Variability of extreme summer precipitation over Circum-BohaiSea region during 1961-2008. Theoretical and applied climatology, v. 104, n. 3-4, p. 501-509, 2011. 
Revista Científica do Corpo de Bombeiros Militar de Pernambuco

XVIII Seminário Nacional de Bombeiros - Foz do Iguaçu PR

Vol.04 N011 - Edição Especial XVIII SENABOM - ISSN 2359-4829

Versão on-line disponível em: http://www.revistaflammae.com.

KNIGHT, C.A \& KNIGHT, N.C. 2001. Severe convective stroms . Americam meteorological society, 28: 223-249.

KOBIYAMA, Masato et al. Prevenção de desastres naturais: conceitos básicos. Curitiba: Organic Trading, 2006.

RONCHAIL, Josyane et al. Inundations in the Mamore basin (south-western AmazonBolivia) and sea-surface temperature in the Pacific and Atlantic Oceans. Journal of Hydrology, v. 302, n. 1-4, p. 223-238, 2005. 